\title{
Imaging to guide ICD therapy: beware what lies beneath
}

\author{
M. T. Rijnierse $\cdot$ P. Knaapen
}

Published online: 4 September 2014

(C) The Author(s) 2014. This article is published with open access at Springerlink.com

The introduction of the implantable cardioverterdefibrillator (ICD) for primary prevention of sudden cardiac death has led to an important reduction in mortality in patients with impaired left ventricular ejection fraction (LVEF) [1, 2]. Consequently, current clinical guidelines recommend ICD implantation for primary prevention in patients with an LVEF below 30-40\% [3]. Although the landmark trials upon which these guidelines are based have utilised various imaging modalities to assess LVEF, two-dimensional (2D) echocardiography has been most frequently used to enrol patients. Furthermore, in clinical practice 2D echocardiography is still most commonly performed for this purpose, as it is inexpensive and readily available. In recent years, however, cardiovascular magnetic resonance imaging (CMR) has emerged as the preferred modality to quantify left ventricular volumes and LVEF due to its high reproducibility and accuracy. As such, CMR is often referred to as the gold standard for LVEF assessment [4]. The question arises, whether these imaging modalities are interchangeable for evaluating the eligibility of ICD therapy. This issue becomes particularly relevant in patients with borderline LVEF values

M. T. Rijnierse $\cdot$ P. Knaapen

Departments of Cardiology and Institute for Cardiovascular Research (IcaR-VU), VU University Medical Center, Amsterdam, the Netherlands

\section{P. Knaapen $(\square)$}

Department of Cardiology, VU University Medical Center,

De Boelelaan 1117, PO Box 7057, 1081 HV Amsterdam, the Netherlands

e-mail: p.knaapen@vumc.nl
(30-40\%) for primary prevention of sudden cardiac death when LVEF is virtually the only parameter to guide such a decision.

The study performed by De Haan et al. published in the current issue of the Netherlands Heart Journal included 152 patients who were referred for primary prevention ICD implantation [5]. All patients underwent LVEF assessment by 2D echocardiography as well as CMR within three months prior to device implantation. De Haan et al. report significantly lower values of left ventricular volumes by $2 \mathrm{D}$ echocardiography, which on average yielded a higher LVEF of $6.6 \%$. Although this percentage might sound trivial, from a clinical point of view such an apparent small difference can have great consequences (see Fig. 1). In fact, when taking the most utilised LVEF cut-off value of $35 \%$ by 2D echocardiography to determine device eligibility, $28 \%$ of patients were reclassified when CMR was used as a reference. Apparently, the choice of imaging has a substantial impact on the decision process of selecting appropriate patients for ICD implantation. Obviously, this raises concern and leads to the question which modality to choose. When stakes are that high for the care of our patients, knowledge on the background and characteristics of such imaging modalities becomes imperative.

The lack of uniformity between imaging modalities to assess LVEF has been well documented. Although comparative studies have shown some discrepancies, most of the data suggest that LVEF tends to be highest when utilising 2D echocardiography and subsequently gradually declines with CMR and nuclear ventriculography, respectively [6]. The quality of echocardiography heavily relies on an adequate acoustic window and bi-plane Simpson's delineation of the endocardial contours may not be obtained in as many as $30 \%$ of 


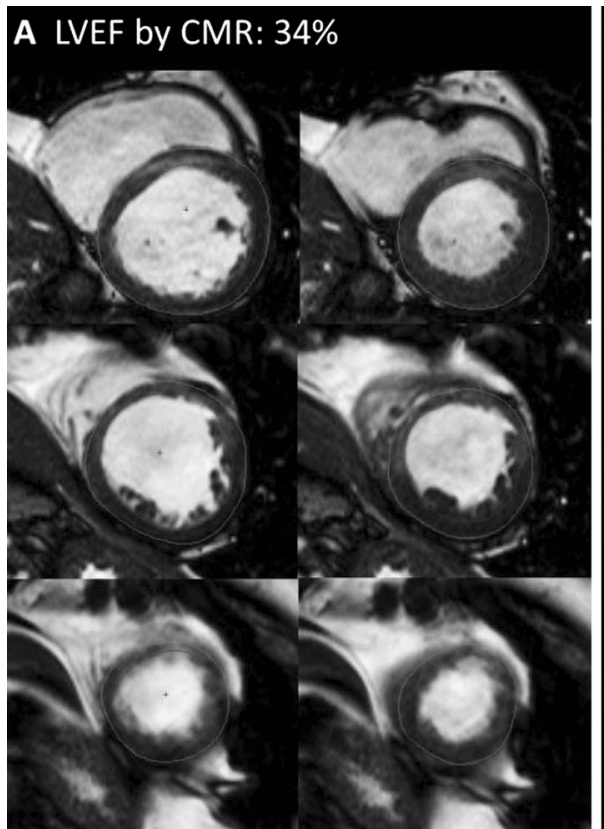

Fig. 1 A 67-year-old patient with ischaemic cardiomyopathy who was screened for ICD implantation for primary prevention of sudden cardiac death. No arrhythmias were documented and the patient did not show signs of heart failure. Both 2D echocardiography and CMR were performed, which yielded a difference in LVEF of $10 \%$ (44 vs. $34 \%$,

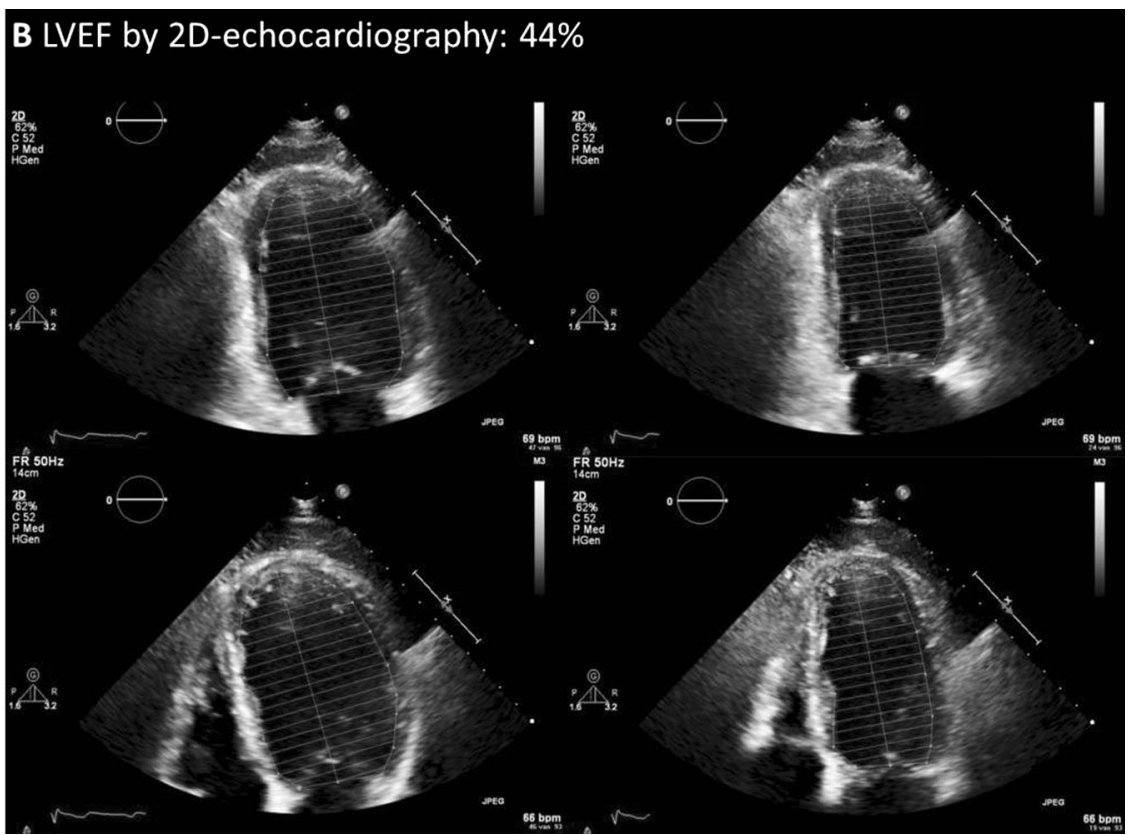

respectively). Based on echocardiographic analysis, ICD implantation should not have been performed, yet CMR drove the decision to implant the device. To date, 2 years after implantation, no ventricular arrhythmic events have occurred

predominantly based on studies using $2 \mathrm{D}$ echocardiography, it could be argued that this type of imaging should be preferred to practice evidence-based medicine. Indeed, the ACC/AHA/ESC 2006 guidelines for ICD implantation actually recommend echocardiography for the assessment of left ventricular function [3]. It is of interest to note that the more recent ACC/AHA/HRS 2008 guidelines have nuanced this recommendation and no longer specify an imaging modality to assess LVEF, as long as it is '... the most clinically accurate and appropriate in their institution' [9]. The current data from De Haan et al. remind us that different imaging modalities should certainly not be considered interchangeable and we should be aware what lies beneath the obtained results of a certain requested test to guide the clinical course of our patients. Even though it is clear that CMR holds great potential to act as gatekeeper for ICD therapy, caution on its interpretation is warranted. Future studies should focus on the clinical consequences of CMR based evaluation of ICD eligibility for primary prevention of sudden cardiac death.

Funding None.

Conflict of interests None declared.
So what should our practice be in the meantime? As current clinical guidelines for ICD implantation are 
Open Access This article is distributed under the terms of the Creative Commons Attribution License which permits any use, distribution, and reproduction in any medium, provided the original author(s) and the source are credited.

\section{References}

1. Bardy GH, Lee KL, Mark DB, et al. Amiodarone or an implantable cardioverter-defibrillator for congestive heart failure. N Engl J Med. 2005;352(3):225-37.

2. Moss AJ, Zareba W, Hall WJ, et al. Prophylactic implantation of a defibrillator in patients with myocardial infarction and reduced ejection fraction. N Engl J Med. 2002;346(12):877-83.

3. Zipes DP, Camm AJ, Borggrefe M, et al. ACC/AHA/ESC 2006 guidelines for management of patients with ventricular arrhythmias and the prevention of sudden cardiac death: a report of the american college of cardiology/american heart association task force and the European society of cardiology committee for practice guidelines (writing committee to develop guidelines for management of patients with ventricular arrhythmias and the prevention of sudden cardiac death): developed in collaboration with the European heart rhythm association and the heart rhythm society. Circulation. 2006;114(10):e385-484.

4. McMurray JJ, Adamopoulos S, Anker SD, et al. ESC guidelines for the diagnosis and treatment of acute and chronic heart failure 2012: the task force for the diagnosis and treatment of acute and chronic heart failure 2012 of the European society of cardiology. Developed in collaboration with the heart failure association (HFA) of the ESC. Eur Heart J. 2012;33(14):1787-847.

5. de Haan S, De Boer K, Commandeur J, Beek AM, van Rossum AC, Allaart CP. Assessment of left ventricular ejection fraction in patients eligible for ICD therapy: Discrepancy between cardiac magnetic resonance imaging and 2D-echocardiography. Neth Heart J. 2014. doi:10. 1007/s12471-014-0594-0.

6. Bellenger NG, Burgess MI, Ray SG, et al. Comparison of left ventricular ejection fraction and volumes in heart failure by echocardiography, radionuclide ventriculography and cardiovascular magnetic resonance; are they interchangeable? Eur Heart J. 2000;21(16):1387-96.

7. Grothues F, Smith GC, Moon JC, et al. Comparison of inter study reproducibility of cardiovascular magnetic resonance with twodimensional echocardiography in normal subjects and in patients with heart failure or left ventricular hypertrophy. Am J Cardiol. 2002;90(1): 29-34.

8. Joshi SB, Connelly KA, Jimenez-Juan L, et al. Potential clinical impact of cardiovascular magnetic resonance assessment of ejection fraction on eligibility for cardioverter defibrillator implantation. J Cardiovasc Magn Reson. 2012;14:69.

9. Epstein AE, DiMarco JP, Ellenbogen KA, et al. ACC/AHA/HRS 2008 guidelines for device-based therapy of cardiac rhythm abnormalities: a report of the american college of cardiology/American heart association task force on practice guidelines (writing committee to revise the ACC/AHA/NASPE 2002 guideline update for implantation of cardiac pacemakers and antiarrhythmia devices): developed in collaboration with the American association for thoracic surgery and society of thoracic surgeons. Circulation. 2008;117(21):e350-408. 